\title{
A newly discovered Bordetella species carries a transcriptionally active CRISPR-Cas with a small Cas9 endonuclease
}

Yury V. Ivanov ${ }^{1 *}$, Nikki Shariat ${ }^{2,3+}$, Karen B. Register ${ }^{4 \dagger}$, Bodo Linz ${ }^{1}$, Israel Rivera ${ }^{1}$, Kai Hu' ${ }^{1}$, Edward G. Dudley ${ }^{2}$ and Eric T. Harvill ${ }^{1,5}$

\begin{abstract}
Background: Clustered regularly interspaced short palindromic repeats (CRISPR) and CRISPR-associated genes (cas) are widely distributed among bacteria. These systems provide adaptive immunity against mobile genetic elements specified by the spacer sequences stored within the CRISPR.

Methods: The CRISPR-Cas system has been identified using Basic Local Alignment Search Tool (BLAST) against other sequenced and annotated genomes and confirmed via CRISPRfinder program. Using Polymerase Chain Reactions (PCR) and Sanger DNA sequencing, we discovered CRISPRs in additional bacterial isolates of the same species of Bordetella. Transcriptional activity and processing of the CRISPR have been assessed via RT-PCR.

Results: Here we describe a novel Type II-C CRISPR and its associated genes—cas1, cas2, and cas9-in several isolates of a newly discovered Bordetella species. The CRISPR-cas locus, which is absent in all other Bordetella species, has a significantly lower GC-content than the genome-wide average, suggesting acquisition of this locus via horizontal gene transfer from a currently unknown source. The CRISPR array is transcribed and processed into mature CRISPR RNAs (crRNA), some of which have homology to prophages found in closely related species B. hinzii.

Conclusions: Expression of the CRISPR-Cas system and processing of crRNAs with perfect homology to prophages present in closely related species, but absent in that containing this CRISPR-Cas system, suggest it provides protection against phage predation. The 3,117-bp cas9 endonuclease gene from this novel CRISPR-Cas system is 990 bp smaller than that of Streptococcus pyogenes, the 4,017-bp allele currently used for genome editing, and which may make it a useful tool in various CRISPR-Cas technologies.
\end{abstract}

Keywords: Bordetella pseudohinzii, Type II CRISPR, Cas9, SpyCas9, Bacteria, Genome editing, Protospacer, GC-content, HGT

\section{Background}

Clustered regularly interspaced short palindromic repeats (CRISPR)-Cas (CRISPR-associated) systems serve as an adaptive immune mechanism in prokaryotes that confer protection against bacteriophages and other mobile elements and vectors [1]. A typical CRISPR-cas locus includes a CRISPR array of containing direct repeats (DR) separated

\footnotetext{
*Correspondence: yvi1@psu.edu

${ }^{\dagger}$ Equal contributors

'Department of Veterinary and Biomedical Sciences, Center for Infectious Disease Dynamics, Center for Molecular Immunology and Infectious Diseases, Pennsylvania State University, University Park, W213 Millennium Science Complex, University Park, PA 16802, USA

Full list of author information is available at the end of the article
}

by spacers $(\mathrm{Sp})$ and adjacent cas genes [2]. In response to invading DNA, CRISPRs acquire short fragments of the foreign nucleic acid sequences and insert those as new spacers at the beginning of the CRISPR array, with each spacer flanked on both sides by direct repeat sequences. This acquisition step involves Cas1, Cas2, and Cas9 proteins [3-5]. Cas9, the signature of Type II CRISPR systems [6], is a RNA-guided endonuclease. CRISPR arrays are transcribed and subsequently processed into small individual CRISPR RNAs (crRNA). This "maturation" step of the array precursor requires a trans-activating crRNA (tracrRNA), an endogenous ribonuclease RNase III, and Cas9 [7, 8]; although RNase-III-independent systems exist for some 
bacteria with Type II-C CRISPRs [9]. In Streptococcus pyogenes, one of the most well studied Type II CRISPR-Cas systems, both tracrRNA and crRNA guide the Cas9 endonuclease to a complementary target sequence (protospacer) to mediate a double-stranded DNA break during target interference. For additional specificity and to avoid cutting within the array itself (autoimmunity), RNA-guided Cas9 cleavage requires a protospacer adjacent motif (PAM; in $S$. pyogenes: $5^{\prime}$-NGG-3') flanking the target site. The specifically targeted endonuclease activity of the $S$. pyogenes Type II-A CRISPR-Cas system has allowed for important breakthrough applications in RNA-guided control of gene expression, genome engineering, and genome editing of multiple organisms $[7,10]$. But limitations of this particular system have led to a search for new CRISPR-Cas systems with altered features.

The publicly available CRISPRfinder program [11] identified CRISPR-cas loci in $45 \%(1176 / 2612)$ of the bacterial genomes analyzed, but CRISPR-Cas systems have not been identified within the genus Bordetella. This genus, which is comprised of nine species, is historically subdivided into "classical" and "non-classical" bordetellae. The extensively studied classical bordetellae consist of the three respiratory pathogens: $B$. pertussis and B. parapertussis, the causative agents of "whooping cough" in humans, and B. bronchiseptica, which causes a broad variety of respiratory disease in many different mammals. The non-classical bordetellae are both genotypically and phenotypically different from the classical bordetellae [12]. They consist of the six recently described species: $B$. hinzii, $B$. holmesii, $B$. ansorpii, B. trematum, B. petrii, and B. avium, all of which are only partially characterized [13-17]. While the classical bordetellae are usually associated with respiratory disease, several non-classical species have also been isolated from wound and ear infection, septicemia and endocarditis, predominantly from immunocompromised patients. For example, B. hinzii, which is a respiratory pathogen in poultry [18] and rodents [19], has also been isolated from humans with chronic cholangitis [20], bacteremia [21], or fatal septisemia [22].

We set out to define the sequence diversity within the Bordetella genus and recently published the genome sequences of numerous isolates from several species [23-26]. During these studies, we discovered a novel species that we named Bordetella pseudohinzii (manuscript in preparation). This species is a close relative of $B$. hinzii and naturally infects laboratory-raised mice. B. hinzii and B. pseudohinzii are distinguishable based on substantial divergence in sequence and gene content, as well as the presence of a CRISPR-Cas system that is unique to the genome of B. pseudohinzii. Here, we describe this novel CRISPR-Cas system, demonstrate that it is transcriptionally active and present evidence that it acts as an adaptive immune system against mobile genetic elements, including bacteriophage sequences present in $B$. hinzii. These data suggest that both species have recently shared an ecological niche with phages, which are represented by the prophages in B. hin$z i i$ genomes and the matching spacers in the genome of B. pseudohinzii, and that acquisition of this CRISPR-Cas system protects against those.

\section{Methods}

\section{Bacterial strains and culture conditions}

Bacterial isolates used in this study are described in Additional file 1: Table S1. Cultures used for preparation of DNA were grown at $37{ }^{\circ} \mathrm{C}$ on Bordet-Gengou agar containing $10 \%$ sheep's blood. Stainer-Scholte broth cultures inoculated with colonies from BordetGengou agar and incubated at $37^{\circ} \mathrm{C}$ with shaking were used for RNA purification. Growth in broth culture was monitored periodically by checking optical density values (at $600 \mathrm{~nm}$ wave length).

\section{DNA isolation, $\mathrm{PCR}$, and sequencing}

DNA used for amplification and sequencing of cas genes and the CRISPR array was purified using a commercially available kit (Promega) and was quantified with a Nanodrop 2000 (Thermo Scientific). Primers for amplification of the complete CRISPR array and of the cas 9 , cas 1 , cas 2 , and $16 \mathrm{~S}$ rRNA genes from other B. pseudohinzii isolates (Additional file 2: Table S2) were designed based on the genome sequence of isolate 8-296-03 [GenBank:JHEP01000084]. PCR reactions included $200 \mu \mathrm{M}$ of dNTPs, $0.5 \mu \mathrm{M}$ of each primer, $1.5 \mathrm{mM}$ of $\mathrm{MgCl}_{2}, 2 \mathrm{U}$ of Taq polymerase (Roche), $5.0 \mu \mathrm{l}$ of $10 \times$ Buffer II, $10 \%$ DMSO and 150 ng of purified DNA template in a final volume of $50 \mu$ l. Cycling conditions for the amplification of cas 9 were $95^{\circ} \mathrm{C}$ for $15 \mathrm{~min}$ and 35 cycles of $95{ }^{\circ} \mathrm{C}$ for $30 \mathrm{~s}, 54{ }^{\circ} \mathrm{C}$ for $30 \mathrm{~s}$ and $72{ }^{\circ} \mathrm{C}$ for $3 \mathrm{~min}$, followed by a final elongation step of $72{ }^{\circ} \mathrm{C}$ for $4 \mathrm{~min}$. Cycling conditions used with the remaining primer pairs were identical except that the extension time was shortened from $3 \mathrm{~min}$ to $1 \mathrm{~min}$. PCR amplicons used for sequencing were purified with ExoSAPIT (USB Corporation) and sequenced at the National Animal Disease Center Genomics Unit using Applied Biosystems Big Dye Terminator v3.1 on an Applied Biosystems 3130 XL Genetic Analyzer sequencer.

\section{CRISPR-cas locus annotation and protospacer prediction}

All Bordetella genome sequences available at GenBank were searched for the presence of CRISPR systems using CRISPRFINDER [11]. Predicted spacer sequences were submitted to BLAST search to query the nucleotide collection (nr/nt) and whole-genome shotgun contigs (wgs) databases at NCBI. Because $E$-value is inadequate when using short nucleotide sequences as BLAST queries, we 


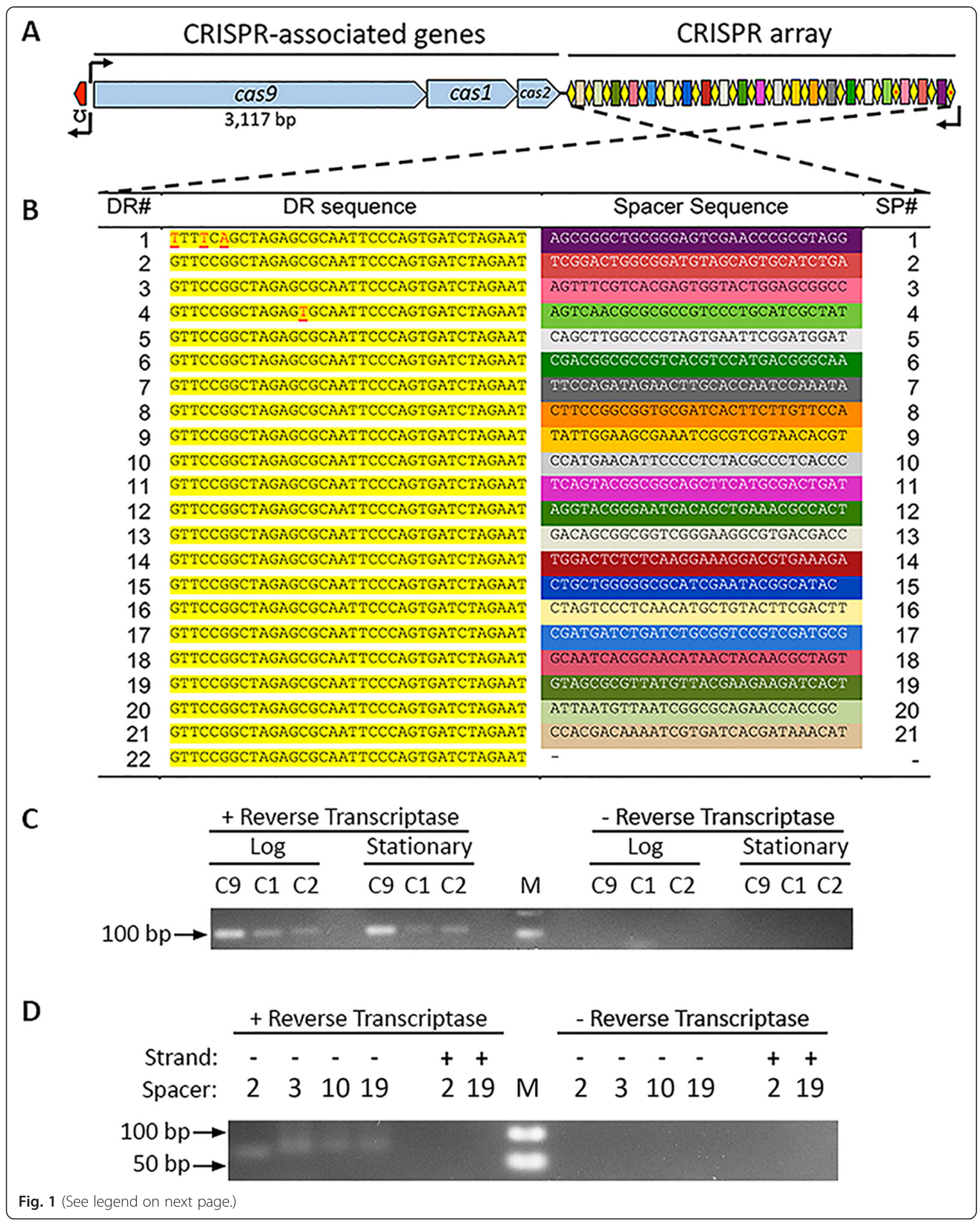


(See figure on previous page.)

Fig. 1 Organization and expression of the Type II-C CRISPR-cas locus of B. pseudohinzii. a Graphical representation of the CRISPR-cas locus. The red block upstream of the cas 9 gene is a putative tracrRNA flanked by the predicted promoter (arrow) and stem-loop terminator (up-side-down sigma symbol). The CRISPR array is enlarged relative to the cas genes for visual clarity. $\mathbf{b}$ Nucleotide sequence of the CRISPR array. SP\# is the spacer sequence number; DR\# is the direct repeat number. Nucleotides deviating from the DR consensus in DR-1 and DR-4 are highlighted in red. c Confirmation of cas9 (C9), cas1 (C1), and cas2 (C2) expression during logarithmic (Log) and stationary phases of growth. Each PCR amplicon was designed to have a similar size. M: 100-bp DNA ladder. d The CRISPR array is processed into individual, mature crRNAs. Positive and negative strands are relative to the orientation shown in Fig. 1a. M: 50-bp DNA ladder

introduce a "percent hit quality" score (\% HQ) to identify and rank the most significant BLAST hits:

$$
\% H Q=\frac{\% \operatorname{cov} \times \% I D}{100 \%}
$$

where \%cov represents the percentage of coverage between the spacer and predicted protospacer sequences and \%ID stands for percent nucleotide identity between the two.

\section{GC-content}

The guanine and cytosine content (GC-content) was calculated within a 120-bp sliding window. The difference in GC-content between the CRISPR-cas locus and the genome average was determined using a two-proportion test implemented in Minitab 17 (www.minitab.com). Briefly, numbers of $\mathrm{G}+\mathrm{C}$ ("positive events") and $\mathrm{A}+\mathrm{T}$ ("negative events") were calculated separately for the chromosome [GenBank:JHEP01000084] and for the CRISPR-cas locus. Because the CRISPR array consists of repetitive sequences, its GC-content is skewed and, therefore, the array sequence was not included. The significance of the difference $(P$-value) was calculated using two-tailed Fisher's exact test.

\section{RNA purification and RT-PCR analysis}

Total RNA was isolated from bacterial cultures during logarithmic growth at $\mathrm{OD}_{600}=0.5$ and during the stationary phase after overnight growth using the TRIzol $^{\oplus}$ Plus RNA Purification System (Life Technologies). To eliminate any residual DNA in the samples, a DNase treatment was implemented during RNA extraction, following the manufacturer's protocol. Reverse transcription reactions were carried out using Superscript III reverse transcriptase (Invitrogen), random hexamer primers and $150 \mathrm{ng}$ of total RNA, following the manufacturer's instructions. Primers for the amplification of cas9, cas1, and cas 2 gene fragments (Additional file 2: Table S2) were designed to yield PCR amplicons of $\sim 100 \mathrm{bp}$ in size (Fig. 1c). The PCR reaction mixture consisted of $2 \mu \mathrm{l}$ of cDNA template $(150 \mathrm{ng} / \mu \mathrm{l})$, $0.2 \mu \mathrm{l}$ of $10-\mathrm{mM}$ dNTP mix, $1 \mu \mathrm{l}$ of $10-\mathrm{mM}$ forward and reverse oligonucleotide primer, $0.2 \mu \mathrm{l}$ of Taq DNA polymerase (1 unit), $2 \mu \mathrm{l}$ of $10 \times$ ThermoPol reaction buffer, and $14.6 \mu \mathrm{l}$ of $\mathrm{ddH}_{2} \mathrm{O}$, in a total volume of $20 \mu \mathrm{l}$. Amplification was carried out at $95{ }^{\circ} \mathrm{C}$ for $10 \mathrm{~min}$, followed by 35 cycles of $95{ }^{\circ} \mathrm{C}$ for $30 \mathrm{~s}, 55^{\circ} \mathrm{C}$ for $30 \mathrm{~s}$, and $72{ }^{\circ} \mathrm{C}$ for $30 \mathrm{~s}$. A final extension step was carried out at $72{ }^{\circ} \mathrm{C}$ for $8 \mathrm{~min}$. PCR products were electrophoresed in $2 \%$ agarose gels and visualized with ethidium bromide under UV-light.

Mature crRNAs were PCR amplified using the QuantiMir RT Kit (Systems Biosciences) following the manufacturer's instructions. Briefly, a poly(A)-tail with an attached adaptor sequence was ligated to the mRNA transcripts, and the product was converted to cDNA. crRNAs corresponding to spacers Sp2, Sp3, Sp10, and Sp19 were PCRamplified from the resulting cDNA library with primers (Additional file 2: Table S2) complementary to the attached adaptor and the individual spacer sequences. The PCR only yielded amplicons from mature crRNAs but not from the unprocessed transcript of the CRISPR array. Each PCR product consisted of a spacer sequence, a flanking part of the direct repeat and an attached poly(A)-tail with a universal primer sequence. The expected size of each of the four tested amplicons is $\sim 85 \mathrm{bp}$. PCR products were electrophoresed in $2.8 \%$ agarose gels and visualized with ethidium bromide under UV-light.

\section{Results}

\section{Annotation of the CRISPR-Cas elements and expression in vitro}

The genome of Bordetella pseudohinzii strain 8-296-03 contains three consecutive, apparently co-transcribed, genes (Fig. 1a) that are homologous to cas9, cas1, and cas2 of Alicycliphilus denitrificans (Additional file 3: Figure S1, Additional file 4: Table S3). Upstream of those genes, a putative tracrRNA is encoded divergently, flanked by a putative promoter and a rho-independent stem-loop terminator. Downstream of the cas genes, the CRISPR array contains 22 direct repeats (DR) and 21 spacer sequences (Sp). Of these, 19 direct repeats are identical, one repeat (DR-4) has a single nucleotide polymorphism (SNP), and the terminal direct repeat (DR-1) has 3 SNPs (Fig. 1b). While each direct repeat is exactly 36 nucleotides in length, the spacer sequences vary: 19 spacers are 30 nucleotides and two are 29 nucleotides long. The sequence of each spacer is unique. Based on the presence and organization of cas 9 , cas 1 , and cas 2 genes within the operon, we typed this CRISPR-Cas system as Type II-C, according to the classification of the CRISPR-Cas systems established by Makarova et al. [6]. 
A functional CRISPR-Cas system requires expression of the cas genes and the CRISPR array, followed by maturation of individual crRNAs. Therefore, we performed an RT-PCR to test whether the cas genes are transcribed during growth in vitro. Amplicons of cas9, cas 1 , and cas 2 were observed from RNA obtained during both logarithmic and stationary phases of growth (Fig. 1c). Processing of the precursor CRISPR array transcript into mature crRNAs was also confirmed by RT-PCR (Fig. 1d).

To predict putative protospacer targets we submitted each spacer sequence to BLAST search. Table 1 summarizes hits with $>80 \%$ hit quality (HQ). Two spacers, Sp8 and $\mathrm{Sp} 9$, are identical to prophage elements found in $B$. hinzii. Sp16, Sp10, and Sp20 show high HQ (97 \%, 86 \%, and $83 \%$, respectively) with different prophages found in $B$. hinzii and B. bronchiseptica and with a capsid gene of a Microviridae-family phage (subfamily Gokushovirinae), respectively. Spacer Sp13 matches a transposase of the IS3/IS911 family with (90\% HQ). Importantly, several prophages identified as likely sources of spacer elements are not found in the genome of $B$. pseudohinzii 8-296-03 but are present in closely related $B$. hinzii, which appear to lack a CRISPR-Cas system. Collectively, these observations suggest that acquisition of the CRISPR-Cas system by $B$. pseudohinzii conferred CRISPR-mediated protection against these bacteriophages and other mobile genetic elements.

The S. pyogenes Cas9 protein (SpyCas9) contains RuvC-like and $\mathrm{HNH}$ motifs that were shown to be essential for its function [7]. We searched for both these motifs in the corresponding Cas9 from B. pseudohinzii (BpsuCas9) (Additional file 5: Figure S2). The RuvC-like endonuclease motif showed $80 \%$ amino acid (aa) similarity (47\% aa identity) and the HNH motif had $66 \%$ aa similarity (33\% aa identity). In each of the motifs, amino acid residues that are conserved among different type-II Cas 9 proteins and that were shown to be essential for Cas9 function are identical in BpsuCas9.

Targeted cleavage by Cas 9 requires a protopspacer adjacent motif (PAM), a short sequence, which is $5^{\prime}$ NGG-3' in S. pyogenes [7]. We attempted to determine in silico a possible PAM sequence that is recognized by BpsuCas9, but the few available protospacer sequences with a high HQ score (Table 1) limited the number of potential sequence candidates. Although eight predicted protospacers and their flanking sequences are not sufficient to conclusively determine the exact PAM sequence, we propose $5^{\prime}-\mathrm{WGR}^{\prime} 3^{\prime}$ as a potential motif used by BpsuCas9 (Additional file 6: Figure S3).

\section{Additional B. pseudohinzii isolates possess the CRISPR-cas locus}

Eleven other isolates identified as B. pseudohinzii on the basis of their 16S rRNA genes were tested for the presence of a CRISPR-cas locus. PCR using genespecific primers confirmed the presence of cas 9 , cas 1 , and cas 2 in all isolates (Fig. 2a-c). A CRISPR array was also found in all isolates but some variation in size was observed (Fig. 2d). Sequencing of the CRISPR array PCR amplicons revealed that their lengths are affected by the loss of $\mathrm{Sp} 14$ in isolate\#2, of $\mathrm{Sp} 8$ to Sp11 in isolate\#6, and of Sp9 in isolate\#10, consistent with the difference in size observed among amplicons (Fig. 2d and e). Each missing spacer is accompanied by the loss of adjacent direct repeat, so that the overall architecture of the array, (-DR-Sp- $)_{n}$, remains intact. The array sequences from all isolates are otherwise identical to one another (Fig. 2e).

Table 1 Highest-scoring BLASTn hits for spacer sequences

\begin{tabular}{|c|c|c|c|c|c|c|}
\hline \multicolumn{3}{|l|}{ BLASTn } & \multirow[t]{2}{*}{ Sp\# } & \multirow[t]{2}{*}{$\% \mathrm{ID}$} & \multirow[t]{2}{*}{$\% \operatorname{cov}$} & \multirow[t]{2}{*}{$\% \mathrm{HQ}$} \\
\hline Protospacer & Locus tag/name & Organism & & & & \\
\hline Prophage: hypothetical protein & L544_3238 & Bordetella hinzii OH87 BAL007II & 8 & 100 & 100 & 100 \\
\hline \multirow[t]{2}{*}{ Prophage: intergenic region } & L544_1114 & Bordetella hinzii OH87 BAL007II & 9 & 100 & 100 & 100 \\
\hline & L544_1115 & & & & & \\
\hline \multirow[t]{2}{*}{ Prophage: replicative DNA helicase } & L541_4891 & Bordetella hinzii CA90 BAL1384 & 16 & 100 & 97 & 97 \\
\hline & dnaB_2 & & & & & \\
\hline Mobile element of IS3/IS911 family & Transposase & Variovorax paradoxus EPS & 13 & 90 & 100 & 90 \\
\hline Pyruvate carboxyl transferase & C791_4721 & Amycolatopsis azurea DSM 43854 & 6 & 93 & 97 & 90 \\
\hline transfer RNA & tRNA-Gly & Bifidobacterium asteroides PRL2011 & 1 & 93 & 93 & 86 \\
\hline Prophage: Siphovirus gp157 & BB3535 & Bordetella bronchiseptica RB50 & 10 & 93 & 93 & 86 \\
\hline \multirow[t]{2}{*}{ Phage structural component } & KF689531.1 & Gokushovirinae clone BBHD08n1 & 20 & 96 & 86 & 83 \\
\hline & major capsid & & & & & \\
\hline
\end{tabular}




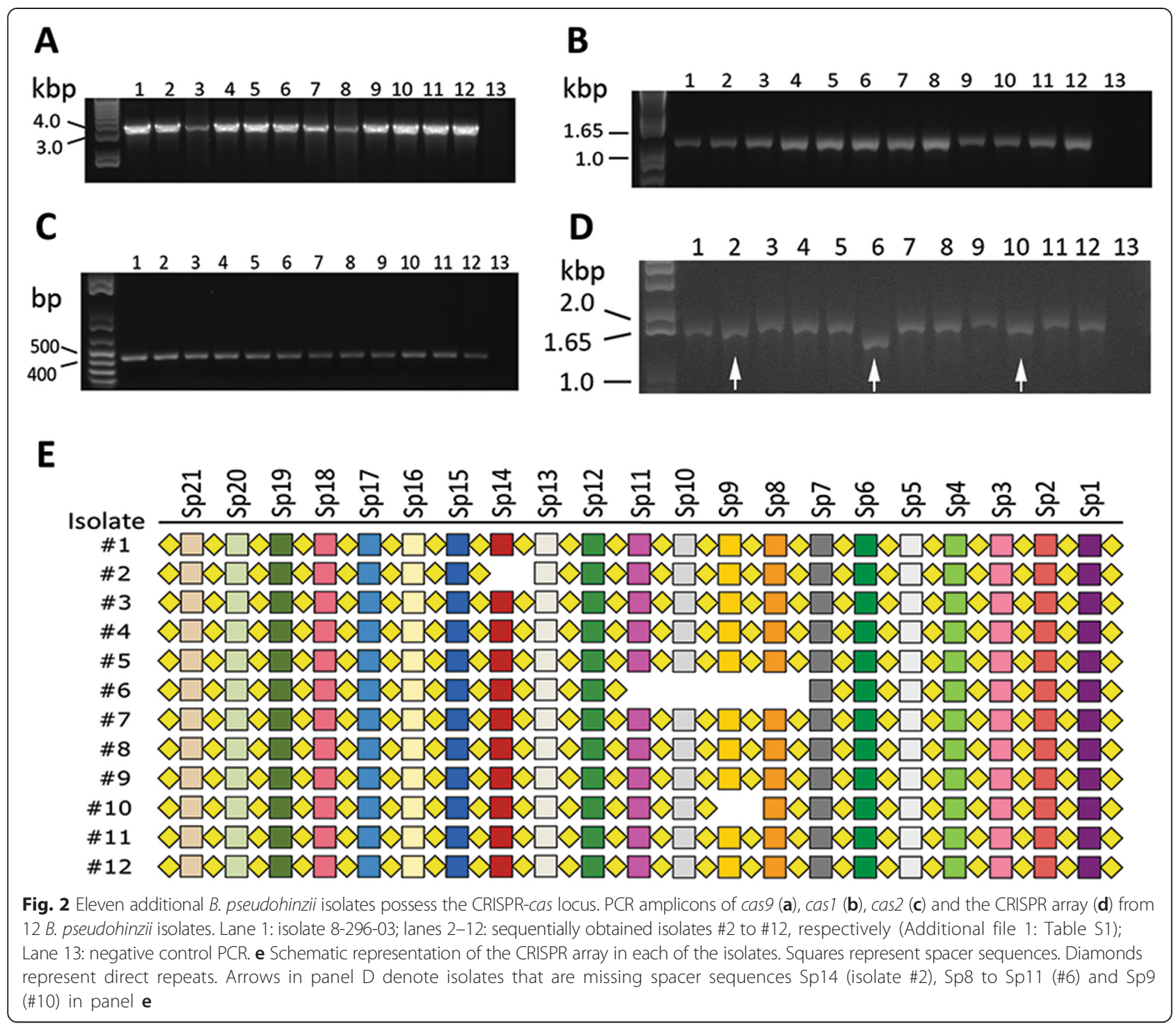

The insertion site of the CRISPR-Cas system is a recombination hotspot in Bordetella

Since we found no evidence for a CRISPR-Cas system in any Bordetella species, suggesting it was acquired solely by the B. pseudohinzii lineage, we assessed the local gene organization near the insertion site of the CRISPRcas locus in other Bordetella genomes (Fig. 3). Only $B$. pseudohinzii and $B$. hinzii exhibit synteny of both the upstream $d a p B-m u r B-t R N A-G l y$ gene cluster and the downstream cluster consisting of a reductase (redoxin) and a disulfide-isomerase $\left(S_{2}\right.$ isom $)$. In other species, synteny is conserved only upstream of the point at which the CRISPR-Cas locus is located in B. pseudohinzii. Genes downstream vary both in identity and orientation, even among isolates of the same species (B. bronchiseptica), suggesting this region is a hotspot for recombination.

Since absence of the CRISPR-Cas system in the other Bordetella species suggests that it was acquired via horizontal gene transfer (HGT), we examined the GC-content of the region including upstream dapB-murB-tRNA-Gly genes, the CRISPR-cas locus, and the downstream redoxin- $S_{2}$ isom genes. The GCprofile of the upstream and downstream genes is consistent with the genome average of $66.5 \%$ (Fig. 4, grey horizontal line). In contrast, the CRISPR-cas locus has a GC-content of $56 \%$, which is significantly lower (twotailed Fisher's exact test, $P<0.01)$. These data strongly suggest that the CRISPR-cas locus of B. pseudohinzii has been horizontally acquired from an unknown source, likely one with a lower GC-content.

Evolutionary relationship and horizontal gene transfer The Cas9 protein is a signature feature of all type-II CRISPR-Cas systems. To identify a possible source of the B. pseudohinzii CRISPR-Cas system, we performed BLAST searches for Cas9 protein sequences (Fig. 5). The 


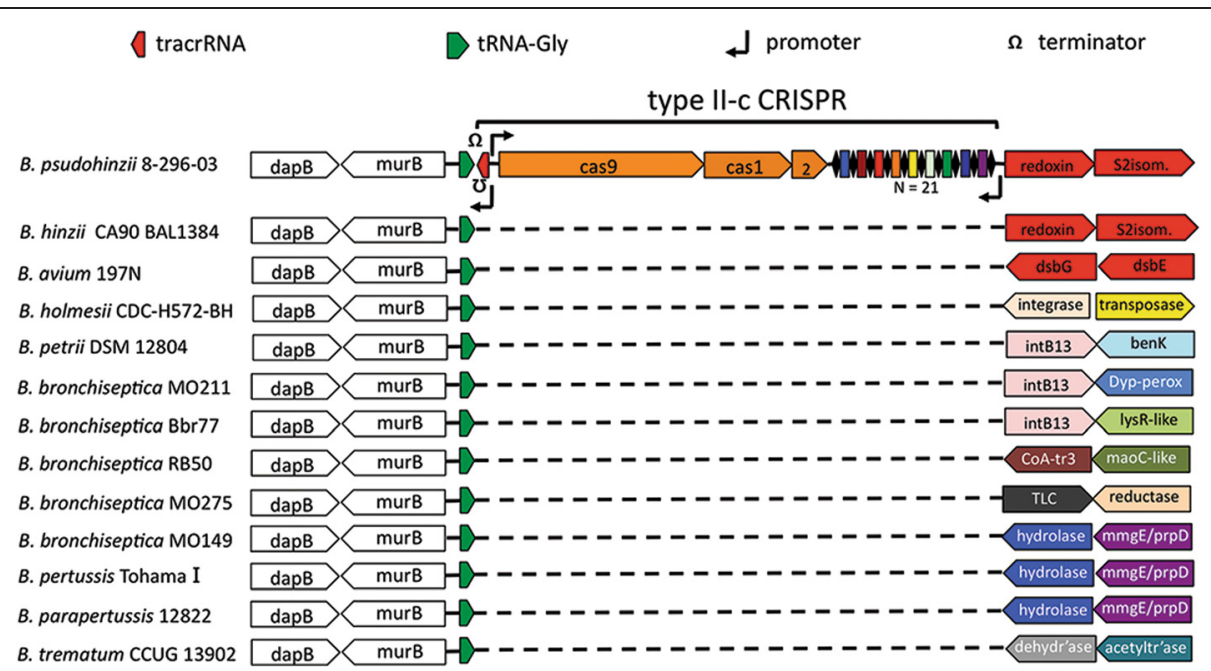

Fig. 3 Local gene organization near the insertion site of the B. pseudohinzii CRISPR-cas locus in multiple Bordetella genomes. The genome synteny is conserved upstream the insertions site, while downstream genes vary in both identity and orientation. Dashed lines denote absence of sequence as compared to B. pseudohinzii 8-296-03. Genes with the same functional annotations are colored identically. The CRISPR array is shown schematically with black diamonds for direct repeats and colored rectangles for spacer sequences; the total number of spacers is 21

two highest-scoring hits, both from Alicycliphilus denitrificans, have $74 \%$ aa identity (Additional file 4: Table S3), suggesting that the proposed recent acquisition of this CRISPR-Cas system into the genome of B. pseudohinzii was probably from an unknown vector. The Cas9-based phylogeny depicted in Fig. 5 includes the highest-scoring hits together with a subset of selected Cas9 sequences previously published elsewhere [27]. Notably, the 5 closest hits are from related genera, all of which belong to the order Burkholderiales in the class Betaproteobacteria. Immediately outside of this clade is the Cas9 from other Betaproteobacteria and from gamma proteobacterium HdN1. The six-member clade of the Burkholderiales,

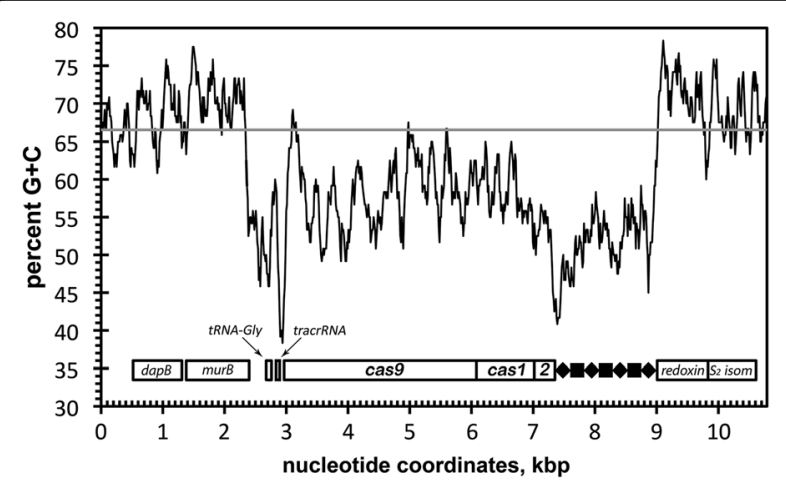

Fig. 4 The GC-content of the B. pseudohinzii 8-296-03 CRISPR-cas locus is significantly lower than that of the genome. The grey horizontal line indicates the average GC-content of $66.5 \%$ for the genome. White rectangles represent genes. The CRISPR array is represented by diamonds ( sequences). On the $x$-axis, 0 corresponds to nucleotide coordinate 24,537 bp of the contig [GenBank:JHEP02000007] including B. pseudohinzii, is not the only occurrence of Cas9 in the Burkholderiales; Ralstonia syzygii and Oligella urethralis also belong to this order but possess divergent Cas9 sequences more closely related to those from a variety of Alphaproteobacteria (Fig. 5). The presence of closely related bacteria within several clades of the tree suggests multiple, independent HGT events associated with the acquisition of CRISPR systems.

To further explore horizontal acquisition of CRISPRCas systems, we calculated the GC-contents for both the cas 9 gene and the genome for all taxa on the tree. The cas 9 sequences ranged from $73.5 \%$ to 29 \% GC-content. Likewise, the genomes varied in a similar range from $70.7 \%$ to $30.5 \%$ GC-content. However, in several cases a discrepancy is apparent between the GC-contents of cas 9 and the corresponding genome (Fig. 5b, column $\Delta$ ). The largest difference was found in $S$. pyogenes M1 whose cas 9 has a GC-content $24.9 \%$ higher than the average for the genome. Discrepancy between the 16SrRNA-gene tree relating bacterial species and the tree relating their cas 9 gene products suggests horizontal acquisition of the CRISPR-Cas. Similarly, GC-content differences between the CRISPR-cas locus and the rest of the genome further support this HGT.

\section{Discussion}

Several lines of evidence suggest that the novel CRISPRCas system described here is functional. We observed active transcription of cas genes and array sequence, as well as maturation of the array transcript. Further, the array contains multiple spacer sequences with homology to prophages in genomes of the most closely related species, $B$. hinzii. Yet, those prophages are absent from $B$. 


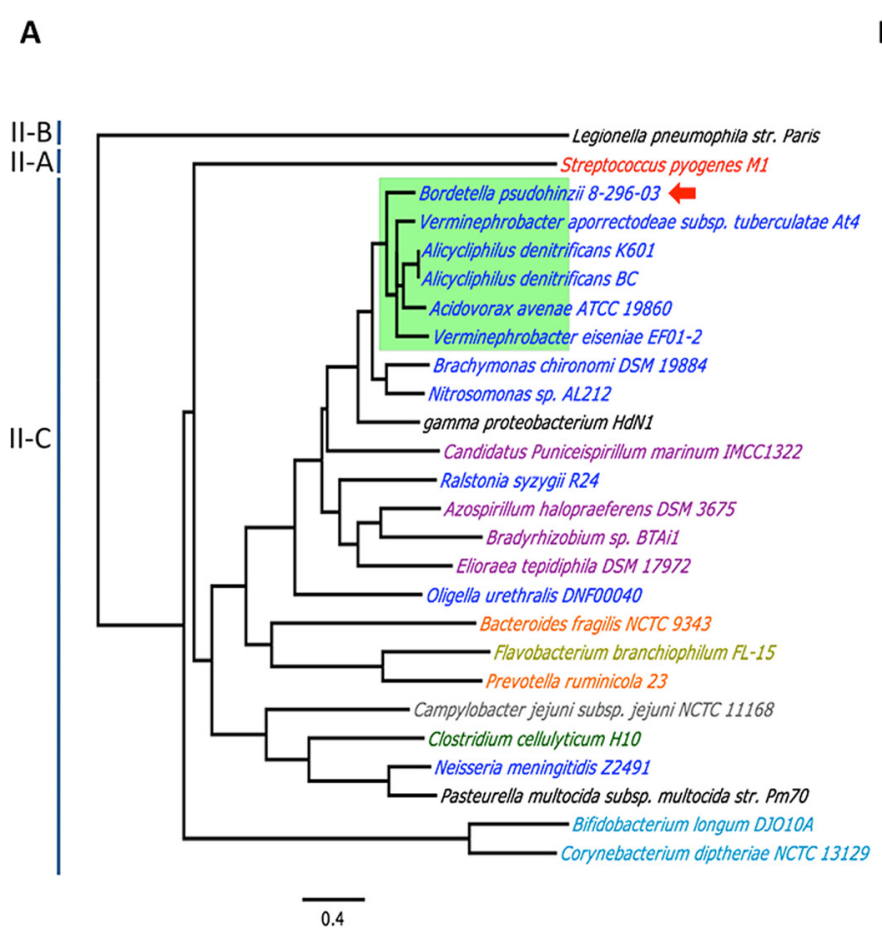

B GC-content (\%)
\begin{tabular}{l|r|r|l} 
cas9 & genome & \multicolumn{1}{|c}{$\Delta$} & + /- \\
\hline 37.3 & 38.4 & 1.1 & - \\
63.4 & 38.5 & 24.9 & + \\
58.3 & 66.5 & 8.2 & - \\
63.3 & 65.7 & 2.4 & - \\
63.4 & 68.0 & 4.6 & - \\
63.4 & 68.3 & 4.9 & - \\
65.6 & 68.8 & 3.2 & - \\
60.8 & 65.3 & 4.5 & - \\
50.1 & 63.6 & 13.5 & - \\
46.8 & 44.8 & 2.0 & + \\
45.9 & 53.3 & 7.4 & - \\
47.9 & 48.8 & 0.9 & - \\
61.9 & 65.9 & 4.0 & - \\
73.5 & 70.7 & 2.8 & + \\
63.4 & 64.9 & 1.5 & - \\
69.6 & 71.3 & 1.7 & - \\
38.7 & 46.2 & 7.5 & - \\
36.7 & 43.2 & 6.5 & - \\
29.2 & 32.9 & 1.3 & - \\
36.2 & 47.7 & 11.5 & - \\
29.0 & 30.5 & 1.5 & - \\
33.2 & 37.4 & 4.2 & - \\
48.9 & 51.8 & 2.9 & - \\
37.6 & 40.4 & 2.8 & - \\
49.9 & 60.1 & 10.2 & - \\
47.9 & 53.5 & 5.6 & - \\
& & &
\end{tabular}

Fig. 5 Cas9-based phylogeny and GC-content of B. pseudohinzii and the highest-scoring BLAST hits. a Maximum likelihood tree based on Cas9 proteins. The green rectangle outlines taxa from the order Burkholderiales. Taxa are colored according to their class-level taxonomic assignment: Gammaproteobacteria in black, Bacilli in red, Betaproteobacteria in blue, Alphaproteobacteria in purple, Bacteroidia in orange, Flavobacteriia in golden, Epsilonproteobacteria in grey, Clostridia in green, Actinobacteria in cyan. All nodes have >50 \% bootstrap support (10,000 replicates). b The GC-content of cas 9 and the corresponding bacterial genome. $\Delta$ is the arithmetic difference between the cas 9 and genome GC-contents; $+/-$ indicates whether cas9 has a lower (-) or higher (+) GC-content

pseudohinzii, suggesting that the CRISPR-Cas may have provided protection against them as an adaptive immune system.

Interestingly, $B$. hinzii contains prophages and $B$. pseudohinzii contains CRISPR-associated spacer sequences that perfectly match those prophages. These observations indicate that both species have been predated by the same phage and have survived that predation in these two different ways. Acquisition of the prophage or the CRISPR-Cas system, either of which would prevent further phage predation, could have also accelerated the divergent evolution of $B$. hinzii and $B$. pseudohinzii by differently affecting uptake or loss of various other genes, contributing to the observed differences in gene content of these closely related species.

It is often observed that horizontally acquired DNA has a lower $\mathrm{GC}$-content than the genome that receives it [28]; and the GC-content of the CRISPR-Cas system in B. pseudohinzii follows this trend. However, our comparison of multiple genomes revealed several cases in which the GC-content of the acquired CRISPR-Cas system is higher than the genome average (Fig. 5). The most striking example is $S$. pyogenes whose cas 9 gene is functional and is successfully used in genome manipulations. This gene has a $25 \%$ higher GC-content than the genome that contains it, suggesting that S. pyogenes acquired its CRISPR-Cas system by HGT and that substantial differences in GC-content do not prevent the function of the Cas 9 protein.

Recent advances in genome editing, genome engineering, and transcriptional control of genes in multiple organisms take advantage of the endonuclease SpyCas9. However, an important limitation of SpyCas9 is its size. The $S$. pyogenes cas 9 allele measures 4,107 base pairs, a size that stretches the carrying capacities of some commonly employed vectors. To address this problem, a recent paper described the use of a 3,159-bp gene encoding Cas9 from Staphylococcus aureus (SaCas9), which recognizes a different PAM sequence $\left(5^{\prime}\right.$ NNGRR-3') [29]. We introduce BpsuCas9, which is of a similarly small size (3,117 bp) and employs a PAM consensus sequence that putatively consists of 5 '-WGR-3' (Additional file 6: Figure S3) and may provide further flexibility with regards to designing guide RNAs. Future experiments will determine the specific features of the B. pseudohinzii CRISPR-Cas system and its potential utility as an additional or alternative tool for genome editing and other applications. 


\section{Conclusions}

This study revealed for the first time presence of the CRISPR-Cas system within the genus Bordetella, in a genome of newly discovered $B$. pseudohinzii sp. nov. We confirmed that this CRISPR-Cas system is actively transcribed and its crRNAs are processed during bacterial growth. Importantly, the CRISPR array carries spacer sequences matching bacteriophages that infect this and two most closely related $B$. hinzii species, thus, conferring adaptive immunity in $B$. pseudohinzii against these phages. The GC-content analysis of the CRISPR-cas locus and homology searches of Cas 9 protein sequences explained how single species of Bordetella acquired this system horizontally from yet an unknown source. The most important observation made about this Bordetella CRISPR-Cas system is its Cas9 endonuclease that is different both in sequence and size from the endonucleases commonly employed in the CRISPR-Cas technology. While the smaller size of BpsuCas9 is of potential utility for more efficient use of biological shuttle vectors during transformations and viral transductions, the unique sequence of BpsuCas9 might allow for some alternative uses of these endonucleases, for example and in addition to the genome editing and genome engineering.

\section{Availability of supporting data}

The data set supporting the results of this article is available in the GenBank repository, [GenBank:JHEP00000000.2] at http://www.ncbi.nlm.nih.gov.

\section{Additional files}

\section{Additional file 1: Table S1. Bacterial isolates used in this study.} (DOC $39 \mathrm{~kb}$ )

Additional file 2: Table S2. Oligonucleotide primer sequences used in this study. (DOC $49 \mathrm{~kb}$ )

Additional file 3: Figure S1. Top three homologous loci encoding $\operatorname{cas} 9$, cas 1 , and $\operatorname{cas} 2$. Top panel, with gene annotations, represents the cas9-cas1-cas2 locus of B. pseudohinzii 8-296-03 (query sequence). Bottom panel summarizes top three BLASTn hit results and illustrates their corresponding alignments against the query. Genome GenBank numbers are shown in blue, above each alignment. (DOC 136 kb)

Additional file 4: Table S3. BLASTp comparisons of Type II Cas proteins. (DOC $77 \mathrm{~kb}$ )

Additional file 5: Figure S2. RuvC-like and HNH-motifs in SpyCas9 and BpsuCas9. RuvC-like motif residue Asp10 and HNH motif residue His840, which are essential for endonuclease activity, are shown in red. Underlined residues are highly conserved among Cas9 proteins from different bacterial species. An * (asterisk) indicates positions at which residue are identical. $\mathrm{A}$ : (colon) indicates positions at which residues are of strongly similar properties. A . (period) indicates conservation between residues of weakly similar properties. (DOC 24 kb)

Additional file 6: Figure S3. Signatures of protospacer adjacent motif (PAM). Vertical lines denote the same nucleotides and not the base pairing between them. Coloring indicates same nucleotides between predicted target sites. (DOC $62 \mathrm{~kb}$ )

\section{Abbreviations}

CRISPR: Clustered regularly interspaced short palindromic repeats; Sp: Spacer sequence; DR: Direct repeat; PAM: Protospacer adjacent motif; crRNA: CRISPR RNA; tracrRNA: Trans-activating CRISPR RNA; HGT: Horizontal gene transfer; SNP: Single-nucleotide polymorphism; GC-content: Guanine + cytosine content; SpyCas9: Cas9 endonuclease of S. pyogenes; SaCas9: Cas9 endonuclease of S. aureus; BpsuCas9: Cas9 endonuclease of B. pseudohinzii; HQ: Hit quality.

\section{Competing interests}

The authors declare that they have no competing interests.

\section{Authors' contributions}

$\mathrm{WI}$ conceived the study, designed and performed experiments and analyses, analyzed the data, and wrote the manuscript; NS and KBR conceived the study, designed and conducted experiments, reviewed and edited the manuscript; BL designed experiments wrote the manuscript; IR designed PCR primers for the CRISPR-Cas, conducted RNA purifications and RT-PCR analyses of crRNA and cas transcripts; $\mathrm{KH}$ : designed and performed GC-content comparisons between cas 9 and the genome; ETH: wrote the edited the manuscript; EGD and ETH conceived and oversaw the study. All authors read and approved the final manuscript.

\section{Acknowledgements}

We thank Ken Boschert for deriving, preserving, and providing B. pseudohinzii isolates for this study; Brian Faddis for additional information and helpful discussions; Liliana Losada, Lauren Brinkac, and JCVI staff for sequencing the genome of B. pseudohinzii. We thank William Boatwright for excellent technical assistance and David Alt, Lea Ann Hobbs and Allen Jensen at the NADC Genomics Unit for DNA sequence data. We thank ARS Culture (NRRL) Collection for preserving bacterial isolates used in this study. The study was supported by National Institutes of Health grants GM083113, Al107016, Al116186, GM113681 (to E.T.H.)

\section{Disclaimer}

Mention of trade names or commercial products in this publication is solely for the purpose of providing specific information and does not imply recommendation or endorsement by the U.S. Department of Agriculture or Pennsylvania State University.

\section{Author details}

${ }^{1}$ Department of Veterinary and Biomedical Sciences, Center for Infectious Disease Dynamics, Center for Molecular Immunology and Infectious Diseases, Pennsylvania State University, University Park, W213 Millennium Science Complex, University Park, PA 16802, USA. ²Department of Food Science, Center for Infectious Disease Dynamics, Center for Molecular Immunology and Infectious Diseases, Pennsylvania State University, University Park, PA 16802, USA. ${ }^{3}$ Present address: Department of Biology, Gettysburg College, Gettysburg, PA 17325, USA. ${ }^{4}$ USDA, Agricultural Research Service, National Animal Disease Center, Ames, IA 50010, USA. ${ }^{5}$ Lee Kong Chian School of Medicine and Singapore Centre on Environmental Life Sciences Engineering, Nanyang Technological University, Singapore 637551, Singapore.

Received: 7 July 2015 Accepted: 6 October 2015

Published online: 26 October 2015

\section{References}

1. Wiedenheft B, Sternberg SH, Doudna JA. RNA-guided genetic silencing systems in bacteria and archaea. Nature. 2012;482(7385):331-8.

2. Barrangou R. The roles of CRISPR-Cas systems in adaptive immunity and beyond. Curr Opin Immunol. 2015;32:36-41.

3. Heler R, Samai P, Modell JW, Weiner C, Goldberg GW, Bikard D, et al. Cas9 specifies functional viral targets during CRISPR-Cas adaptation. Nature. 2015:519(7542):199-202.

4. Yosef I, Goren MG, Qimron U. Proteins and DNA elements essential for the CRISPR adaptation process in Escherichia coli. Nucleic Acids Res. 2012;40(12):5569-76.

5. Nunez JK, Kranzusch PJ, Noeske J, Wright AV, Davies CW, Doudna JA. Cas1-Cas2 complex formation mediates spacer acquisition during CRISPR-Cas adaptive immunity. Nat Struct Mol Biol. 2014;21(6):528-34.

6. Makarova KS, Haft DH, Barrangou R, Brouns SJ, Charpentier E, Horvath P, et al. Evolution and classification of the CRISPR-Cas systems. Nat Rev Microbiol. 2011;9(6):467-77. 
7. Jinek M, Chylinski K, Fonfara I, Hauer M, Doudna JA, Charpentier E. A programmable dual-RNA-guided DNA endonuclease in adaptive bacterial immunity. Science. 2012;337(6096):816-21.

8. Deltcheva E, Chylinski K, Sharma CM, Gonzales K, Chao Y, Pirzada ZA, et al. CRISPR RNA maturation by trans-encoded small RNA and host factor RNase III. Nature. 2011:471(7340):602-7.

9. Zhang Y, Heidrich N, Ampattu BJ, Gunderson CW, Seifert HS, Schoen C, et al. Processing-independent CRISPR RNAs limit natural transformation in Neisseria meningitidis. Mol Cell. 2013;50(4):488-503.

10. Sander JD, Joung JK. CRISPR-Cas systems for editing, regulating and targeting genomes. Nat Biotechnol. 2014;32(4):347-55.

11. Grissa I, Vergnaud G, Pourcel C. CRISPRFinder: a web tool to identify clustered regularly interspaced short palindromic repeats. Nucleic Acids Res. 2007;35(Web Server issue):W52-7.

12. Gross R, Keidel K, Schmitt K. Resemblance and divergence: the "new" members of the genus Bordetella. Med Microbiol Immunol. 2010;199(3):155-63.

13. Gross R, Guzman CA, Sebaihia M, dos Santos VA, Pieper DH, Koebnik R, et al. The missing link: Bordetella petrii is endowed with both the metabolic versatility of environmental bacteria and virulence traits of pathogenic bordetellae. BMC Genomics. 2008:9:449-2164-9-449.

14. Pittet LF, Emonet S, Schrenzel J, Siegrist CA, Posfay-Barbe KM. Bordetella holmesii: an under-recognised Bordetella species. Lancet Infect Dis. 2014;14(6):510-9.

15. Temple LM, Weiss AA, Walker KE, Barnes HJ, Christensen VL, Miyamoto DM, et al. Bordetella avium virulence measured in vivo and in vitro. Infect Immun. 1998:66(11):5244-51.

16. Vandamme P, Heyndrickx M, Vancanneyt M, Hoste B, De Vos P, Falsen E, et al. Bordetella trematum sp. nov., isolated from wounds and ear infections in humans, and reassessment of Alcaligenes denitrificans Ruger and Tan. Int J Syst Bacteriol 1996. 1983:46(4):849-58.

17. Ko KS, Peck KR, Oh WS, Lee NY, Lee JH, Song JH. New species of Bordetella, Bordetella ansorpii sp. nov., isolated from the purulent exudate of an epidermal cyst. J Clin Microbiol. 2005:43(5):2516-9.

18. Register KB, Sacco RE, Nordholm GE. Comparison of ribotyping and restriction enzyme analysis for inter- and intraspecies discrimination of Bordetella avium and Bordetella hinzii. J Clin Microbiol. 2003;41(4):1512-9.

19. Jiyipong T, Morand S, Jittapalapong S, Raoult D, Rolain JM. Bordetella hinzii in rodents. Southeast Asia Emerg Infect Dis. 2013;19(3):502-3.

20. Arvand M, Feldhues R, Mieth M, Kraus T, Vandamme P. Chronic cholangitis caused by Bordetella hinzii in a liver transplant recipient. J Clin Microbiol. 2004:42(5):2335-7.

21. Cookson BT, Vandamme P, Carlson LC, Larson AM, Sheffield JV, Kersters K, et al. Bacteremia caused by a novel Bordetella species, "B. hinzii". J Clin Microbiol. 1994;32(10):2569-71.

22. Kattar MM, Chavez JF, Limaye AP, Rassoulian-Barrett SL, Yarfitz SL, Carlson LC, et al. Application of 165 rRNA gene sequencing to identify Bordetella hinzii as the causative agent of fatal septicemia. J Clin Microbiol. 2000;38(2):789-94

23. Harvill ET, Goodfield LL, Ivanov Y, Meyer JA, Newth C, Cassiday P, et al. Genome sequences of 28 Bordetella pertussis U.S. outbreak strains dating from 2010 to 2012. Genome Announc. 2013;1(6): doi:10.1128/genomeA.01075-13.

24. Harvill ET, Goodfield LL, Ivanov Y, Smallridge WE, Meyer JA, Cassiday PK, et al. Genome sequences of nine Bordetella holmesii strains isolated in the United States. Genome Announc. 2014;2(3):doi:10.1128/genomeA.00438-14.

25. Register KB, Ivanov YV, Harvill ET, Brinkac L, Kim M, Losada L. Draft genome sequences of six Bordetella hinzii isolates acquired from avian and mammalian hosts. Genome Announc. 2015;3(2):doi:10.1128/ genomeA.00081-15.

26. Register KB, Ivanov YV, Jacobs N, Meyer JA, Goodfield LL, Muse SJ, et al. Draft genome sequences of 53 genetically distinct isolates of Bordetella bronchiseptica representing 11 terrestrial and aquatic hosts. Genome Announc. 2015;3(2):doi:10.1128/genomeA.00152-15.

27. Chylinski K, Makarova KS, Charpentier E, Koonin EV. Classification and evolution of type II CRISPR-Cas systems. Nucleic Acids Res. 2014;42(10):6091-105.

28. Nishida H. Genome DNA sequence variation, evolution, and function in bacteria and archaea. Curr Issues Mol Biol. 2012;15(1):19-24.

29. Ran FA, Cong L, Yan WX, Scott DA, Gootenberg JS, Kriz AJ, et al. In vivo genome editing using Staphylococcus aureus Cas9. Nature. 2015;520:186-91.

\section{Submit your next manuscript to BioMed Central and take full advantage of:}

- Convenient online submission

- Thorough peer review

- No space constraints or color figure charges

- Immediate publication on acceptance

- Inclusion in PubMed, CAS, Scopus and Google Scholar

- Research which is freely available for redistribution

Submit your manuscript at www.biomedcentral.com/submit 